International Journal of Bifurcation and Chaos

(c) World Scientific Publishing Company

\title{
Phase Space Structure and Transport in a Caldera Potential Energy Surface
}

\author{
MATTHAIOS KATSANIKAS AND STEPHEN WIGGINS \\ School of Mathematics, University of Bristol, University Walk, Bristol BS8 1TW, UK \\ matthaios.katsanikas@bristol.ac.uk,s.wiggins@bristol.ac.uk \\ Received (to be inserted by publisher)
}

\begin{abstract}
We study phase space transport in a 2D caldera potential energy surface (PES) using techniques from nonlinear dynamics. The caldera PES is characterized by a flat region or shallow minimum at its center surrounded by potential walls and multiple symmetry related index one saddle points that allow entrance and exit from this intermediate region. We have discovered four qualitatively distinct cases of the structure of the phase space that govern phase space transport. These cases are categorized according to the total energy and the stability of the periodic orbits associated with the family of the central minimum, the bifurcations of the same family, and the energetic accessibility of the index one saddles. In each case we have computed the invariant manifolds of the unstable periodic orbits of the central region of the potential and the invariant manifolds of the unstable periodic orbits of the families of periodic orbits associated with the index one saddles. The periodic orbits of the central region are, for the first case, the unstable periodic orbits with period 10 that are outside the stable region of the stable periodic orbits of the family of the central minimum. In addition, the periodic orbits of the central region are, for the second and third case, the unstable periodic orbits of the family of the central minimum and for the fourth case the unstable periodic orbits with period 2 of a period-doubling bifurcation of the family of the central minimum. We have found that there are three distinct mechanisms determined by the invariant manifold structure of the unstable periodic orbits govern the phase space transport. The first mechanism explains the nature of the entrance of the trajectories from the region of the low energy saddles into the caldera and how they may become trapped in the central region of the potential. The second mechanism describes the trapping of the trajectories that begin from the central region of the caldera, their transport to the regions of the saddles, and the nature of their exit from the caldera. The third mechanism describes the phase space geometry responsible for the dynamical matching of trajectories originally proposed by Carpenter and described in Collins et al. 2014 for the two dimensional caldera PES that we consider.
\end{abstract}

Keywords: Chaos and Dynamical Systems, Chemical Reaction Dynamics, Chemical Physics

\section{Introduction}

In this paper we analyze the phase space structure and transport in a $2 \mathrm{D}$ caldera like potential energy surface (PES). The caldera PES is characterized by a flat region or shallow minimum at its center surrounded by potential walls and multiple symmetry related index one saddle points that allow entrance and exit from this intermediate region. This shape of the potential resembles the collapsed region within an 
errupted volcano (caldera), and this is the reason that Doering Doering et al., 2002] and co-workers refer to this type of potential as a caldera.

A caldera PES arises in many organic chemical reactions, such as the vinylcyclopropane-cyclopentene rearrangement Baldwin, 2003, Goldschmidt et al., 1968, the stereomutation of cyclopropane Doubleday et al., 1997, the degenerate rearrangement of bicyclo[3.1.0] hex-2-ene [Doubleday et al., 1999, 2006 1 or 5methylenebicyclo[2.1.0]pentane Reyes et al. 2002]. A key feature of this class of PES is that the stationary point corresponding to the intermediate often possesses higher symmetry than those of either the reactant or product stationary points. Under such circumstances, conventional statistical kinetic models would predict that the symmetry of the intermediate should be expressed in the product ratio. However, in the examples cited this is not observed, and it is the desire to understand this fact that has made analysis of the dynamics of reactions occuring on caldera potentials particularly important Carpenter et al., 2016; Doering et al. 1968. Fig. 1 shows the potential energy contours of a two dimensional caldera studied in Collins et al. Collins et al., 2014. In this model the symmetry-related 1-index saddles points provide a configuration space mechanism allowing for the the entrance of trajectories into and exit from the intermediate region of the caldera. Examining Fig. 1, one can easily suppose that the caldera is a decision point or crossroads in the reaction where selectivity between the four points of the saddles, that are indicated by black points, is determined. The subject of this paper is to investigate the phase space structure and transport associated mechanisms of the decision in the caldera and the origin of the selectivity between the four regions of the saddles. In order to address these questions Collins et al. Collins et al., 2014] performed a detailed study of the trajectories in this two dimensional model. Broadly speaking, they identified two types of trajectory behaviour: dynamical matching [Collins et al., 2014] and trajectory trapping in the caldera.

In this paper, we analyze the phase space mechanisms and the origin of the trajectory behaviour that was found in Collins et al., 2014. For this reason we study the phase space structure of the caldera and we use many methods from the theory of dynamical systems in order to have a deep insight of the dynamics of the system. We have computed all of the basic families of periodic orbits of the system and the invariant objects around them. We have found the mechanisms that are responsible for the entrance and the exit from the caldera and the phase space mechanisms for trapping and "dynamical matching". This could not have been achieved without the study of the phase space structure since these mechanisms are based on the geometrical objects in the phase space (invariant manifolds of the unstable periodic orbits).

The structure of the paper is the following. In section 2 we describe the mathematical model for our system and the method of the surface of section that we used for the study of the phase space structure, and the families of the periodic orbits. In section 3 we describe our results for the four different cases of the structure of the phase space that we discovered in our study. Then we present the summary and our conclusions in section 4. For completeness we give an appendix A describing how we characterize the linear stability of periodic orbits and their bifurcations and an appendix B that describes the computation and characterization of periodic orbit dividing surfaces.

\section{Model and Methods}

In this section we describe the 2D autonomous Hamiltonian system that is a model for the dynamics on the caldera. We introduce the method of Poincaré surface of section used to investigate the phase space structure of our Hamiltonian System and we also present the families of periodic orbits of our system.

\subsection{Hamiltonian system and equations of motion}

The Caldera potential has been used in a previous study from [Collins et al. 2014]. As we described in the 1, the topography of the potential is similar with the collapsed region of an erupted volcano. It has at the center a shallow minimum where there is a stable equilibrium point, the central minimum (see Table 2.1. The shallow minimum of the potential is surrounded by potential walls. On these potential walls we have the existence of four 1-index saddles (two for lower values of Energy and other two for higher values of Energy, see Table 2.1) that allow the entrance into and exit from the intermediate region of the caldera. The four regions of the saddles are the only regions where molecules can enter into and exit from the caldera. The caldera has a stable equilibrium point at the center (that is represented by a black point 


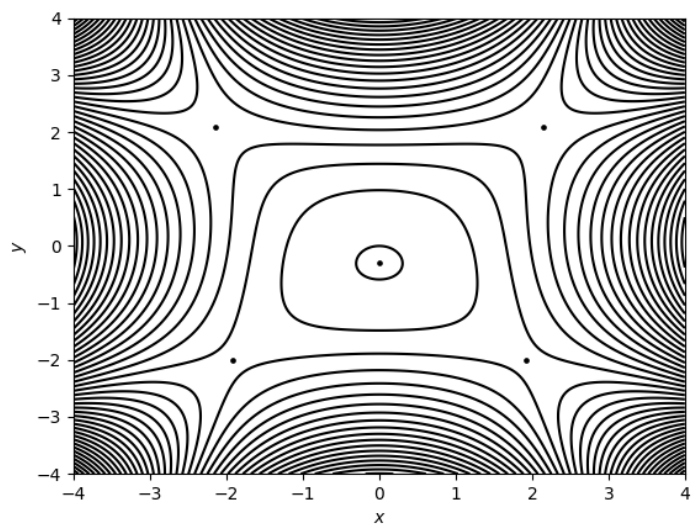

Fig. 1. The stationary points (depicted by black points) and contours of the potential.

at the center of the Fig. 1p and it is like a decision point in the reaction between four different paths that lead to four different regions of saddles and then to the exit from the caldera (that are represented by four black points in the Fig. 11). In addition, the entrance into the caldera is achieved through the four different regions of the saddles. The potential is:

$$
\begin{gathered}
V(x, y)=c_{1} r^{2}+c_{2} y-c_{3} r^{4} \cos [4 \theta]= \\
c_{1}\left(y^{2}+x^{2}\right)+c_{2} y-c_{3}\left(x^{4}+y^{4}-6 x^{2} y^{2}\right)
\end{gathered}
$$

where $r^{2}=x^{2}+y^{2}, \cos [\theta]=x / r$. Potential parameters are taken to be $c_{1}=5, c_{2}=3, c_{3}=-3 / 10$. The potential is symmetric with respect to the y-axis.

The Hamiltonian of the system is:

$$
H\left(x, y, p_{x}, p_{y}\right)=\frac{p_{x}^{2}}{2 m}+\frac{p_{y}^{2}}{2 m}+V(x, y)
$$

with potential $V(x, y)$ in eq. (1) and $m=1$.

The equations of motion are:

$$
\begin{array}{r}
\dot{x}=\frac{p_{x}}{m} \\
\dot{y}=\frac{p_{y}}{m} \\
\dot{p}_{x}=-\frac{\partial V}{\partial x}(x, y) \\
\dot{p}_{y}=-\frac{\partial V}{\partial y}(x, y)
\end{array}
$$

\subsection{Surfaces of section and Periodic orbits}

In this paper we used the computational method of surfaces of section in order to study the phase space of a Hamiltonian system. The phase space of an autonomous Hamiltonian system with two degrees of freedom is a 4 -dimensional space $\left(x, y, p_{x}, p_{y}\right)$. The energy of the system (the value of the Hamiltonian) is a constant of motion and one variable, for example $p_{y}$ (as we did in this paper), can be derived from the 


\begin{tabular}{llll}
\hline Critical point & $\mathrm{x}$ & $\mathrm{y}$ & $\mathrm{E}$ \\
\hline Central minimum & 0.000 & -0.297 & -0.448 \\
Upper LH saddle & -2.149 & 2.0778 & 27.0123 \\
Upper RH saddle & 2.149 & 2.0778 & 27.0123 \\
Lower LH saddle & -1.923 & -2.003 & 14.767 \\
Lower RH saddle & 1.923 & -2.003 & 14.767 \\
\hline
\end{tabular}

other three variables for fixed values of energy. This means that the phase space of the system is reduced to a 3-dimensional space $\left(x, y, p_{x}\right)$. If we define a particular surface $S\left(x, y, p_{x}, p_{y}\right)=$ const. within every fixed interval of time, then the first intersection $S_{1}$ of an initial point $S_{0}$ is called the first return of $S_{0}$ and the surface $S$ as Poincaré surface of section. The returns of the points on $S$, for a fixed value of energy, define a map that is called as Poincaré map [Poincaré, 1892; Birkhoff, 1960]. The surfaces of section help us to relate the study of the phase space of a $2 \mathrm{D}$ autonomous Hamiltonian system to the study of the phase space of a 2D map, the Poincaré map. This allows us a simple visualization of the phase space geometry of the system. In all cases we used the Poincaré surfaces of section $y=\operatorname{const}\left(2 \mathrm{D}\right.$ space $\left.\left(x, p_{x}\right)\right)$ with $p_{y}>0$ that is computed from the other coordinates for a fixed value of energy. On a 2D Poincaré map the phase space areas are conserved [Poincaré, 1892].

Every solution of a system like this of (2) is periodic if it repeats itself after time $\mathrm{T}$, that is called the period. For example, If we define a trajectory in the phase space as $\left(x(t), y(t), p_{x}(t), p_{y}(t)\right)$ ( $\mathrm{t}$ is the time), we can denote the initial conditions of a periodic orbit as $\left(x_{p}(0), y_{p}(0), p_{x_{p}}(0), p_{y_{p}}(0)\right)$ at time $\mathrm{t}=0$. After time $T$ (the period of the periodic orbit) we will have that $\left(x_{p}(0), y_{p}(0), p_{x_{p}}(0), p_{y_{p}}(0)\right)$ $=\left(x_{p}(T), y_{p}(T), p_{x_{p}}(T), p_{y_{p}}(T)\right)$. A periodic orbit is a one-dimensional closed curve in the phase space. A periodic orbit of a Hamiltonian system of two degrees of freedom is represented by at least one fixed point on the 2D Poincaré surface of section before closing. The periodic orbits that are represented by more than one point, $n>1$ points, are periodic orbits with high order multiplicity and we will refer to them as periodic orbits with period $n$ or as n-periodic orbits. For example a periodic orbit with period 10 (10-periodic orbit) is represented by 10 points on the 2D Poincaré section.

According to the theory of linear stability of periodic orbits in autonomous Hamiltonian systems with two degrees of freedom, we have two types of periodic orbits; the stable and unstable periodic orbits. In the case of the stable periodic orbits we have the existence of 2-dimensional invariant tori (KAM invariant tori) surrounding them, according the KAM theorem [Kolmogorov, 1954, Arnold, 1963; Moser, 1962]. This means that there are trajectories (quasi-periodic orbits) around the stable periodic orbits that lie forever on these invariant tori. These KAM invariant tori are topological barriers on the energy surface of the 2D autonomous Hamiltonian systems. As a consequence, trajectories inside the tori are trapped inside forever and trajectories outside the tori can never enter the tori. On the contrary, the unstable periodic orbits repel the trajectories in their neighbourhood.

We can understand better the different trajectory behaviour in the neighbourhood of the two types of periodic orbits with an example. In this example, we study the trajectory behaviour in the neighbourhood of stable and unstable periodic orbits. This can be achieved if we apply a small perturbation to the initial conditions of a periodic orbit. In our example we used the periodic orbits of the family of the central minimum. The periodic orbits of this family are represented by vertical straight lines in the configuration space (see as example the periodic orbit for $\mathrm{E}=17$ at Fig. 2). If we perturb the stable periodic orbits of the family for $\mathrm{E}=20$ and 33 the resulting trajectories are very close to the vertical straight line of the periodic orbit (Fig. 3). But if we perturb the unstable periodic orbit for $\mathrm{E}=29$ we observe that the resulting trajectory diverges from the vertical straight line of the periodic orbit (Fig. 3). As we see, the range of the deviation from the periodic orbit does not depend on the value of the energy but on the kind of linear stability of periodic orbits (stable or unstable). In addition, we observe that the trajectories for $E=20$ and 33 are represented by invariant curves on the surfaces of section (Fig 3 ). This happens because as we explained above the trajectories in the neighbourhood of the stable periodic orbits lie on 2-dimensional tori in the phase space that are represented by invariant curves on the $2 \mathrm{D}$ surfaces of section. In the case of a trajectory in the neighbourhood of the unstable periodic orbit (for $E=29$ ), the trajectory goes far 

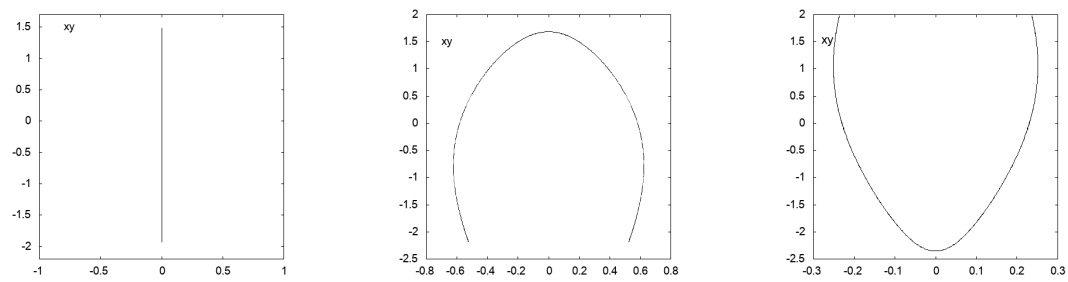

Fig. 2. The periodic orbits of the family of the central minimum and its bifurcations in the configuration space. Left panel: The periodic orbit of the family of the central minimum for $\mathrm{E}=17$. Central panel: The periodic orbit of the first period-doubling bifurcation of the family of the central minimum for $\mathrm{E}=23$. Right panel: The periodic orbit of the second period-doubling bifurcation of the family of the central minimum for $\mathrm{E}=30$.
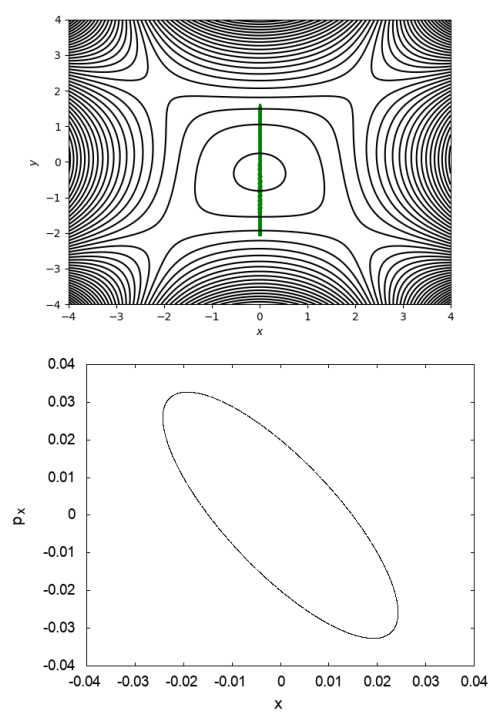
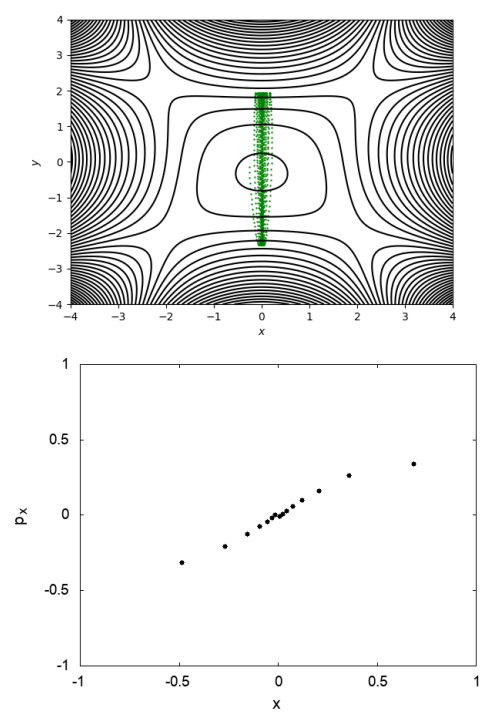
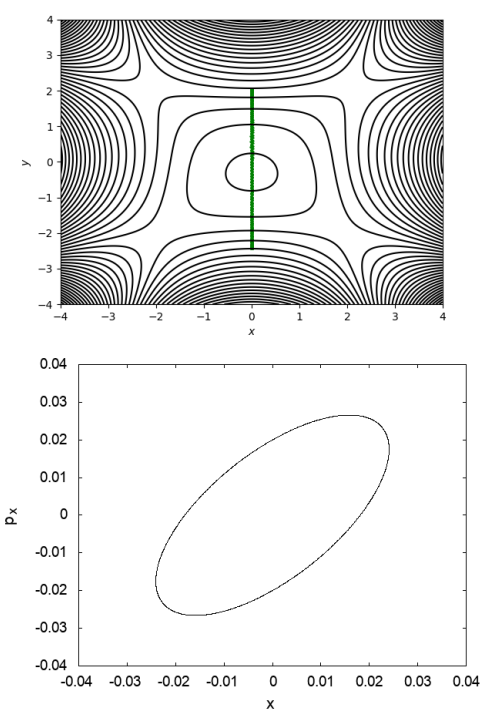

Fig. 3. The resulting trajectories if we perturb (with $\Delta p_{x}=0.02$ and $t=20$ time units ) the periodic orbits of the family of periodic orbits of the central minimum for values of Energy $\mathrm{E}=20$ (upper left panel), $\mathrm{E}=29$ (upper central panel) and $\mathrm{E}=33$ (upper right panel). We also depict the corresponding Poincaré surfaces of section for $\mathrm{E}=20$ (lower left panel), E=29 (lower central panel) and $\mathrm{E}=33$ (lower right panel).

away from the periodic orbit and this behaviour is represented on the 2D surfaces of section (Fig. 3). The method of the computation and the mathematical description of the linear stability of periodic orbits is given in appendix A.

It is known [Poincaré, 1892] that if we start with a particular periodic orbit of a system and then we vary continuously a parameter of the system we find a set of periodic orbit that is called a family. If we apply the Lyapunov subcenter theorem Rabinowitz et al., 1982, Weinstein, 1973; Moser, 1976] at the case of the stable equilibrium point, we have the existence of at least two families of periodic orbits that are associated with the equilibrium point (central minimum). We computed the two families that are associated with the central minimum and their bifurcations for our system. By means of a standard continuation method (using the Newton-Raphson algorithm) we computed the two families on the plane $y=0$. The initial conditions $\left(x, y, p_{x}, p_{y}\right)$, on the plane $y=0$, for the one family is $\left(0,0,0, p_{y}>0\right)$ and for the other is $\left(0,0,0, p_{y}<0\right)$. In Fig. 4 , we give the diagram of the x-coordinate (on the plane $\left.y=0\right)$ of these families and their bifurcations versus energy. We depict the stable and unstable parts of the families of periodic orbits with red and cyan color, respectively.

The families of periodic orbits of the central minimum are initially stable but then they become unstable, at the value for the energy $\mathrm{E}=21$ (Fig. 4). At this point, we have a new supercritical perioddoubling bifurcation (see appendix A) for each of the two families. This means that we have a new family of periodic orbits with double period than that of the parent family. The new family has two symmetric 


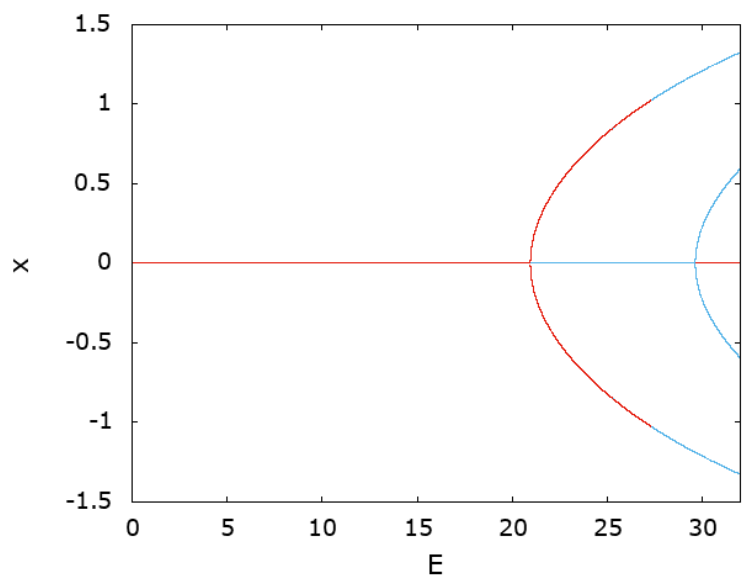

Fig. 4. The diagram of the $\mathrm{x}$-coordinate of the initial conditions on the plane $y=0$, for the families of periodic orbits that start from the central minimum and their bifurcations, versus energy. The red and cyan parts of the families indicate the stable and unstable parts of the families of periodic orbits, respectively.

branches that are represented by two symmetric arches in Fig. 4 . This family is initially stable and then it becomes unstable for larger value of the energy (Fig. 4).

After the bifurcation point, the families of periodic orbits of the central minimum become again stable for $\mathrm{E}=29.64$ (see Fig, 4). At this point, we have a new subcritical period-doubling (see appendix A) bifurcation, for each of two families, that is unstable (Fig. 4). We noticed that eventually each of the two families of periodic orbits of the central minimum has two period-doubling bifurcation at the points where we have transition from stability to instability and vice versa. The periodic orbits of the family of the central minimum and their bifurcations are depicted in the configuration space in Fig. 2,

According to the Lyapunov subcenter theorem [Rabinowitz et al., 1982; Weinstein, 1973; Moser, 1976 we have at least one family that is associated with every index 1 saddles of our system. In our case we found two families for every index 1 saddle and we computed them. We computed these families using a standard continuation method (using the Newton-Raphson algorithm) on the planes $y=a$, with $a=-1.8019693$ for the case of the lower energy saddles and $a=1.884090$ for the case of higher energy saddles. The general form of the initial conditions $\left(x, y, p_{x}, p_{y}\right)$ for the one family of saddles is $\left(x_{0}, a, p_{x_{0}}, p_{y}>0\right)$ and for the other is $\left(x_{0}, a,-p_{x_{0}}, p_{y}<0\right)$. In particular, the families of upper right hand (RH) saddles have $x_{0}>0$ and $p_{x_{0}}<0$, the families of upper left $(\mathrm{LH})$ hand saddles have $x_{0}<0$ and $p_{x_{0}}>0$, the families of lower right hand $(\mathrm{RH})$ saddles have $x_{0}>0$ and $p_{x_{0}}>0$ and the families of Lower left (LH) hand saddles have $x_{0}<0$ and $p_{x_{0}}<0$. These families are unstable. The periodic orbits of the families of the saddles are depicted in the configuration space in Fig. 5.

In all cases, we studied the cases of periodic orbits with positive values $p_{y}$. The results are similar for the case of the periodic orbits with negative values $p_{y}$.

\section{Results}

In this section we investigate the different kinds of trajectories that enter into and exit from the caldera using the notion of dividing surfaces. Then we study the phase space structure in order to understand the origin of the trajectory behaviour and the mechanisms that support this behaviour. For this reason, we use the notion of Poincaré section and we compute the invariant manifolds of the unstable periodic orbits.

We encountered four different cases for the trajectory behaviour and the phase space structure of our model. These cases are:

(1) Case I: In this case the periodic orbits of the families of the central minimum are stable. As we can see in Fig. 4, these families are initially stable and continue as stable for values of Energy until $E=21$. For these values of energy we have also the unstable periodic orbits of the families of low energy saddles.

(2) Case II: In this case the periodic orbits of the families of the central minimum are unstable after the 

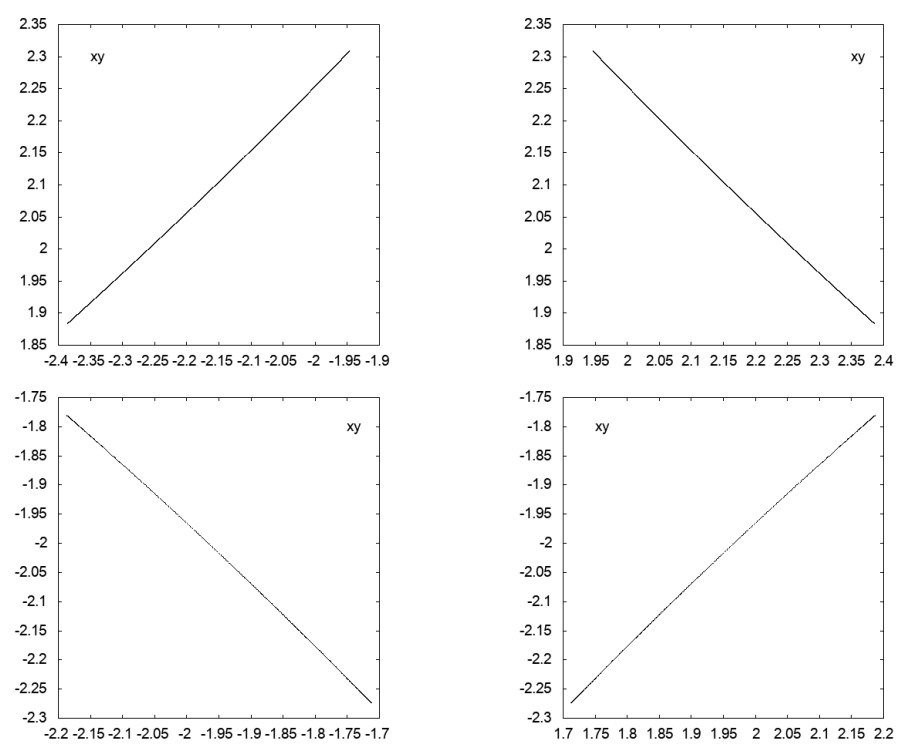

Fig. 5. The periodic orbits of the families of saddles in the configuration space. Upper left panel: The periodic orbit of the family of the upper left hand saddle for $\mathrm{E}=29$. Upper right panel: The periodic orbit of the family of the upper right hand saddle for $\mathrm{E}=29$. Lower left panel: The periodic orbit of the family of the lower left hand saddle for $\mathrm{E}=17$. Lower right panel: The periodic orbit of the family of the lower right hand saddle for $\mathrm{E}=17$.

first period-doubling bifurcation of these families (for values of Energy larger than $\mathrm{E}=21$ - see Fig. 4). Moreover, we consider the interval of energy before the appearance of high energy saddles (for $\mathrm{E}=27.0123)$. For these values of energy we have also the unstable periodic orbits of the families of low energy saddles.

(3) Case III : In this case the periodic orbits of the families of the central minimum are unstable but they exist after the appearance of high energy saddles (for $\mathrm{E}=27.0123$ ) and before the second perioddoubling bifurcation of these families (for $\mathrm{E}=29.64$ ). For these values of energy we have also the unstable periodic orbits of the families of high and low energy saddles.

(4) Case IV: In this case, the periodic orbits of the families of the central minimum are stable. This case is after the second period-doubling bifurcation of these families (for $\mathrm{E}=29.64$ - see Fig. 4). For these values of Energy we have again the unstable periodic orbits of the families of high and low energy saddles.

In the following sections, we study a representative example for every case.

\subsection{Case I}

In this subsection, we study a representative example for the first case, $\mathrm{E}=17$. The periodic orbits of the central minimum are stable. In Fig. 6 we see that the trajectories that have initial conditions on the dividing surfaces of the periodic orbits of the family of central minimum are trapped for a long time at the central region of the caldera and then they exit from the regions of the lower saddles. In addition, we observe in the same figure that the trajectories that have initial conditions on the dividing surfaces of the periodic orbits of families of the lower saddles are trapped for a long time after the entrance into the caldera and before they exit from it.

We investigated the origin of this trajectory behaviour through the study of the phase space structure. We studied the phase space in the neighborhood of the stable periodic orbit of the central minimum on the Poincaré section $y=0$ with $p_{y}>0$. We see in figure 7 invariant curves around the stable periodic orbit of the central minimum that is represented by one point at the center $(0,0)$. The existence of these curves is guaranteed by the KAM (Kolmogorov-Arnold-Moser) theorem Kolmogorov, 1954; Arnold, 1963; Moser, 1962]. According this theorem there are trajectories that lie on 2-dimensional invariant tori (KAM invariant tori) around the stable periodic orbits in a Hamiltonian system with two degrees of freedom (see 

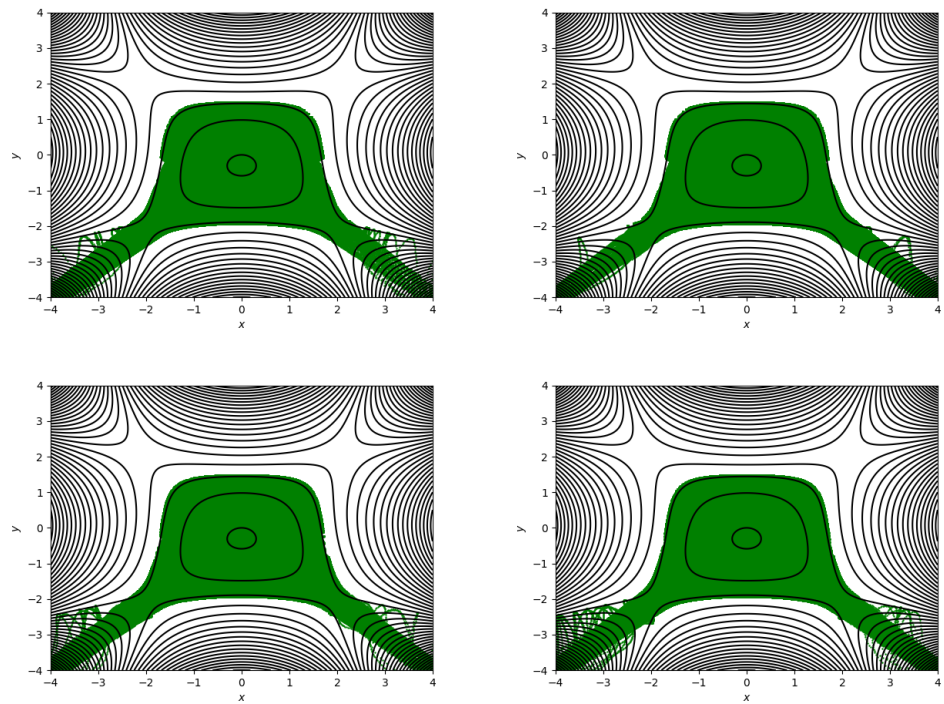

Fig. 6. Upper Left Panel: Trajectories that have initial conditions on the dividing surface of a periodic orbit of the family of periodic orbits of lower left hand saddle for $\mathrm{E}=17$ and contours of the potential energy. Upper Right Panel: Trajectories that have initial conditions on the dividing surface of a periodic orbit of the family of periodic orbits of the lower right hand saddle for $\mathrm{E}=17$ and contours of the potential energy. Lower Left Panel: Trajectories that have initial conditions on the dividing surface of a periodic orbit of the family of periodic orbits of the central minimum for $\mathrm{E}=17$ and contours of the potential energy. Lower Right Panel: Trajectories that have initial conditions on the dividing surface of an unstable periodic orbit with period 10 for $\mathrm{E}=17$ and contours of the potential energy.

also 2.2). These tori are represented by invariant curves on the $2 \mathrm{D}$ Poincaré section like those that we observe around the stable periodic orbit of the central minimum in Fig. 77. This means that the trajectories close to the stable periodic orbit of the central minimum lie on these tori for ever and they are trapped in the central region of the caldera.

In Fig. 7 we see also invariant curves around a stable 6-periodic orbit between the invariant curves around the central stable periodic orbit. All these trajectories are trapped between the tori that are around the stable periodic orbit of the central minimum (see 2.2). This explains that there are trajectories around the central area of the caldera but it cannot explain the transport of trajectories to the regions of lower saddles.

If we observe carefully the upper panel of Fig. 7 we can discern ten small green regions that are outside the invariant curves. We can see by enlargement one of these regions in the lower panel in the Fig. 7 and we observe small green invariant curves. This means that these regions are stable areas and indicate the presence of a stable periodic orbit with period 10. This means that we have also an accompanied unstable periodic orbit with period 10 that is represented by ten black points (upper panel of Fig. 7) . The morphology of this orbit in the configuration space can be seen in Fig. 8.

We computed the invariant manifolds of the unstable periodic orbit with period 10 on the 2D Poincaré section $y=0$ with $p_{y}>0$. The invariant manifolds of a periodic orbit in a Hamiltonian system system with two degrees of freedom are 2D geometrical objects in the energy surface and their existence is guaranteed by the theorem of unstable and stable manifolds Wiggins, 2003. These are 1-dimensional objects on the 2D Poincaré surfaces of section. We have two kinds of invariant manifolds, the unstable manifold and the stable manifold. All trajectories with initial conditions on these objects approach the periodic orbit (stable manifold) and move away from the periodic orbit (unstable manifold).

The computation of the invariant manifolds on the 2D Poincaré section $y=0$ with $p_{y}>0$ has been done numerically. As we know (see appendix A) an unstable periodic orbit has two real eigenvalues of the monodromy matrix of the Poincaré map. The one is inside the unit circle and associated with the stable manifold and the other is outside of the unit circle and associated with the unstable manifold. For the calculation of the unstable and stable manifolds we need to take many initial conditions (in this 

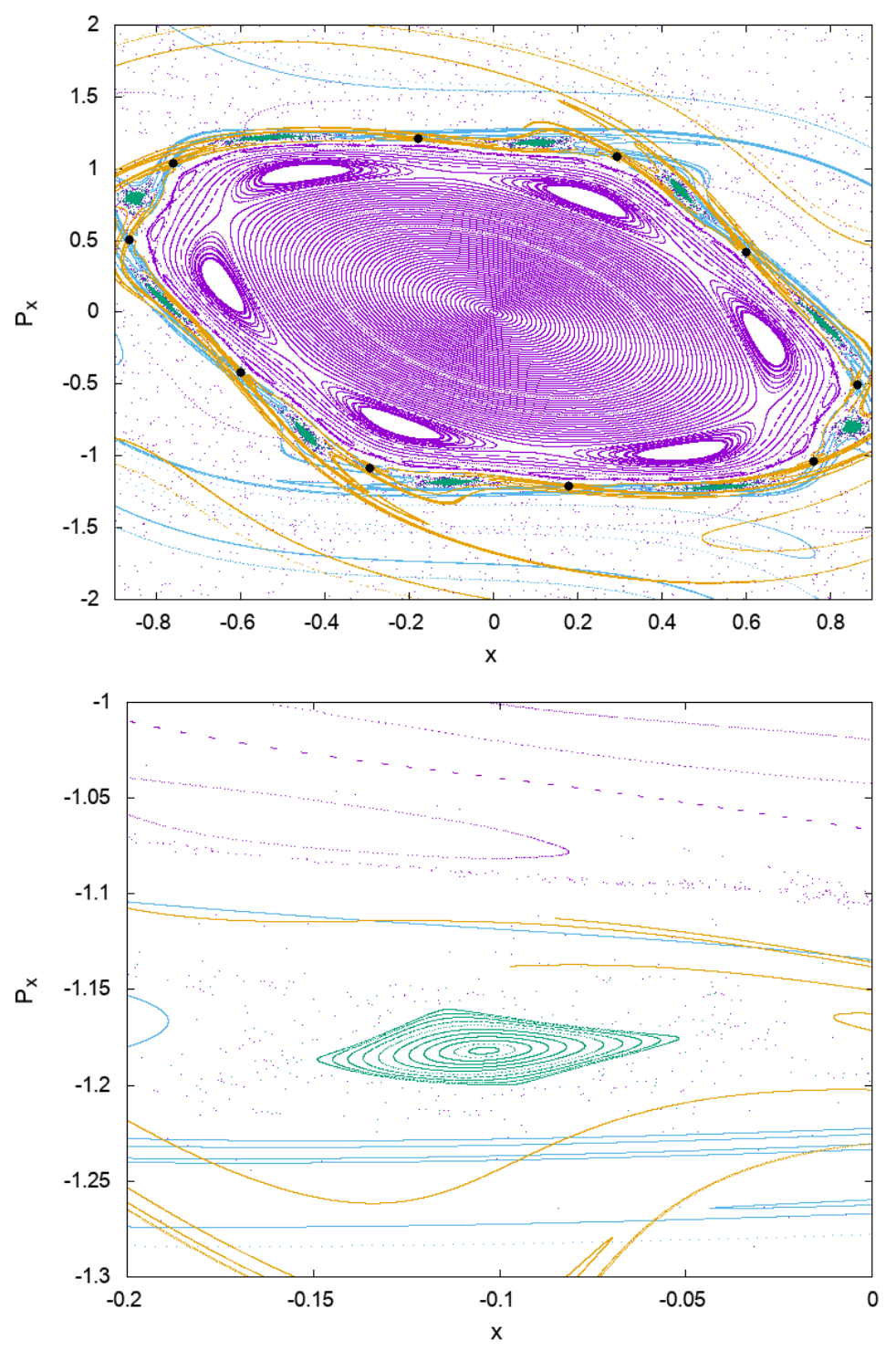

Fig. 7. Upper Panel: Poincaré surface of section $y=0$ with $p_{y}>0$ for $\mathrm{E}=17$. We observe invariant curves around the stable periodic orbit of the family of central minimum. We also observe the unstable (with cyan color) and stable (with orange color) invariant manifolds of an unstable periodic orbit with period 10, that is represented by ten black points. Furthermore, we see the invariant curves (with green color) around the ten points of the accompanied stable periodic orbit with period 10. Lower Panel: An enlargement of the previous figure in order to see the invariant curves (with green color) in one of the ten stable regions that are around the ten points of the stable periodic orbit with period 10 .

paper 20000 ) in the direction of the unstable eigenvector (the eigenvector of the monodromy matrix of the Poincaré map that corresponds to the eigenvalue outside the unit circle) and stable eigenvector (the eigenvector of the monodromy matrix of the Poincaré map that corresponds to the eigenvalue inside the unit circle) and take their consequents and their antecedents respectively.

The unstable and stable manifolds of the periodic orbit with period 10 are depicted by cyan and orange colors in Figs. 7 and 9. We see that the invariant manifolds start from the ten points of the periodic orbit (Fig. 7) and have many oscillations, that is an indication of strong chaos. Then the manifolds extend throughout the whole available space (Fig. 9 ). This is because of the fact that this periodic orbit is outside the central stable region around the stable periodic orbit of the central minimum and there are not restrictions to the manifolds of the periodic orbit to occupy the available space except the space that is occupied from the tori around the stable periodic orbit of the central minimum . Moreover, we can observe in Fig. 9 that many points are trapped in the lobes of the invariant manifolds for long time. This means that 


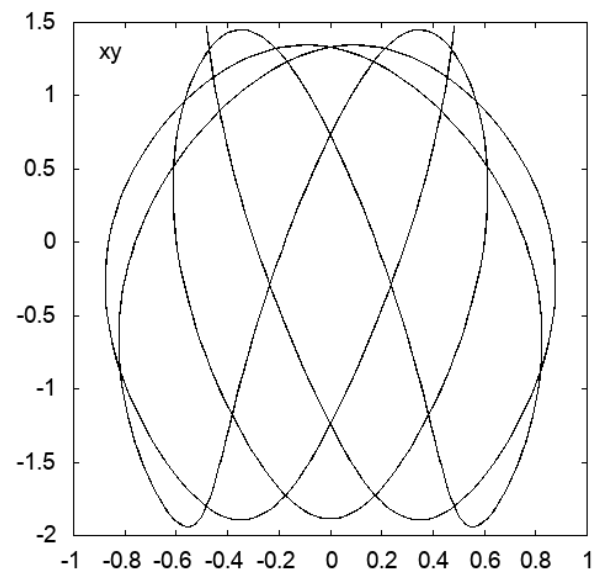

Fig. 8. An unstable periodic orbit with period 10 in the configuration space, for $\mathrm{E}=17$.

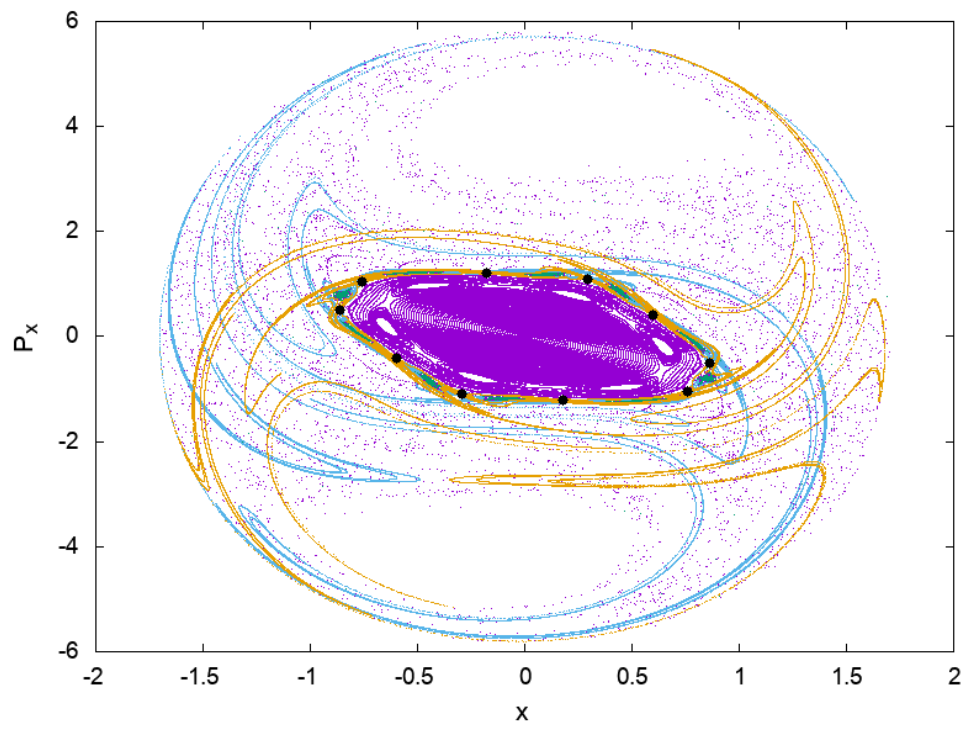

Fig. 9. Poincaré surface of section $y=0$ with $p_{y}>0$ for $\mathrm{E}=17$. We see the extension of the invariant manifolds (unstable with cyan color and stable with orange color) in the whole available space. The central region of the figure is depicted by enlargement of the upper panel of Fig 7 .

there are many trajectories that are trapped by the invariant manifolds of the unstable periodic orbit with period 10. This mechanism explains the trapping of trajectories in the central region of the caldera and the transport of these trajectories to large regions of the phase space. The trajectories in the neighbourhood of an unstable periodic orbit will move away from the periodic orbit following the unstable manifold. If we take initial conditions on the dividing surface of the unstable periodic orbit with period 10, we will observe that the trajectories, after their trapping by the manifolds for long time, exit from the regions of the lower saddles (lower right panel of Fig 6). From this behaviour we conclude that the unstable manifold of the unstable periodic orbit of period 10 guides the trajectories far away from the neighbourhood of the periodic orbit and to the exit from the two regions of lower saddles of the caldera.

As we described above, the invariant manifolds of the unstable periodic orbit with period 10 are responsible for the trapping and the transport of trajectories in the caldera. But, what is the role of unstable periodic orbits of lower saddles? In order to understand this we computed the invariant manifolds of the unstable periodic orbits of the lower saddles on the 2D Poincaré section $y=-1.8019693$ with $p_{y}>0$ (this is the surface of section on which we can see these periodic orbits because they do not cross the plane $y=0$ ). As we can see in Fig. 10, the invariant manifolds are depicted with cyan (for the unstable 


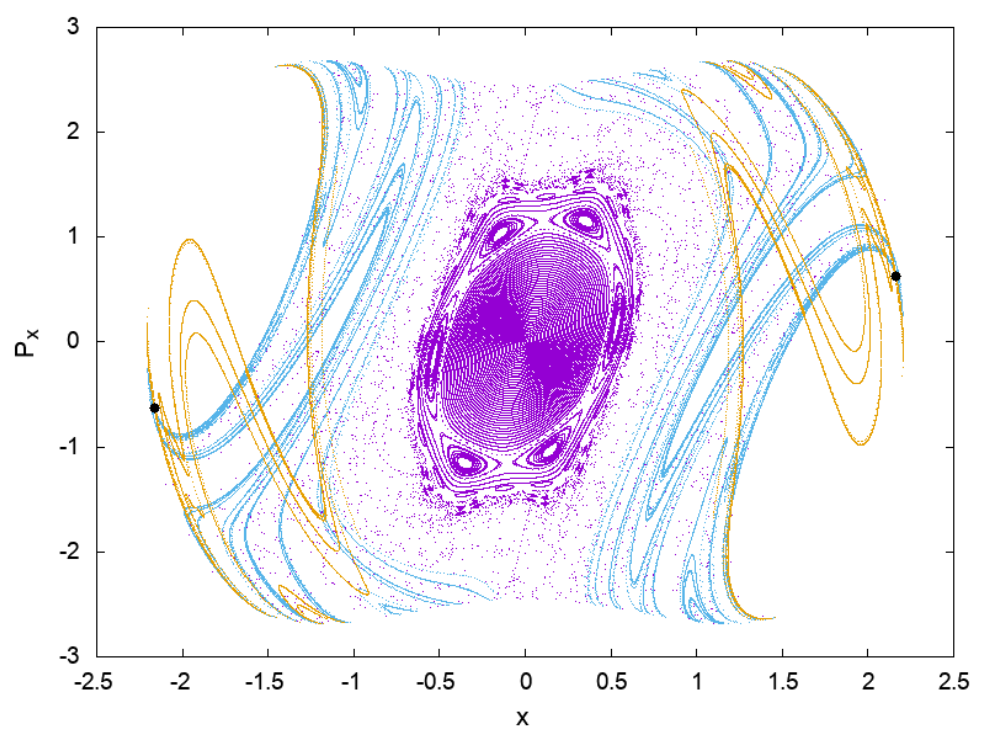

Fig. 10. Poincaré surface of section $y=-1.8019693$ with $p_{y}>0$ for $\mathrm{E}=17$. We see the unstable (cyan color) and stable (orange color) invariant manifolds of the periodic orbits of two lower saddles, that are represented by two black points. Furthermore, we observe invariant curves around the stable periodic orbit of the central minimum and cloudy points outside from them.

manifold) and orange (for the stable manifold). The invariant manifolds extend into the central region which is occupied from the invariant curves around the stable periodic of the central minimum and many scattered points that are outside of them. We observe also that many points are trapped in the lobes of the invariant manifolds. This means that many trajectories are transported from the unstable manifold of the periodic orbits of the lower saddles far away from the periodic orbits towards the central region of the caldera. Then the trajectories are trapped by the invariant manifolds of the unstable periodic orbit with period 10 as we can see in Fig. 9. The trajectories in the neighbourhood of an unstable periodic orbit approach the periodic orbit following the stable manifold. This means that many trajectories, that are trapped, are guided from the stable manifold to the central region of the caldera which is the region of the unstable periodic orbit with period 10. This explains the fact that the trajectories that have initial conditions on the dividing surfaces of the periodic orbits of the lower saddles enter the central region of the caldera (see upper right and left panels of Fig. 6).

\subsection{Case $I I$}

In this case the periodic orbits of the central minimum have become unstable and we have the appearance of new stable periodic orbits with period 2 after the bifurcation of the family of the central minimum. We study a representative example for $\mathrm{E}=23$. The trajectories that have initial conditions on the dividing surfaces of the periodic orbits of the family of the central minimum, its bifurcation and families of lower saddles have similar behaviour like this that we observed in the case I (see Fig. 6).

In order to study the origin of this trajectory behaviour we studied the phase space structure for $\mathrm{E}=23$. In Fig. 11, we see that there are no invariant curves, on the Poincaré section $y=0$ with $p_{y}>0$, around the periodic orbit of the family of the central minimum, but scattered collection of points. Moreover, we observe invariant curves around a stable periodic orbit with period 2 . This periodic orbit belongs to the bifurcation of the central family of the central minimum (lower panel of Fig 11). We have many trajectories that lie on the tori in the phase space (invariant curves on the Poincaré section) and they are trapped forever in the central region around the stable periodic orbit with period 2. Furthermore, we observe many points on the Poincare section (Fig. 11) inside the invariant manifolds of the unstable periodic orbit of the central minimum. This has as a consequence that many trajectories are trapped by the invariant manifolds of the periodic orbit of the central minimum and they are transported from the unstable invariant manifold far away from the periodic orbit and finally to the exit from the caldera. This is the same mechanism as 

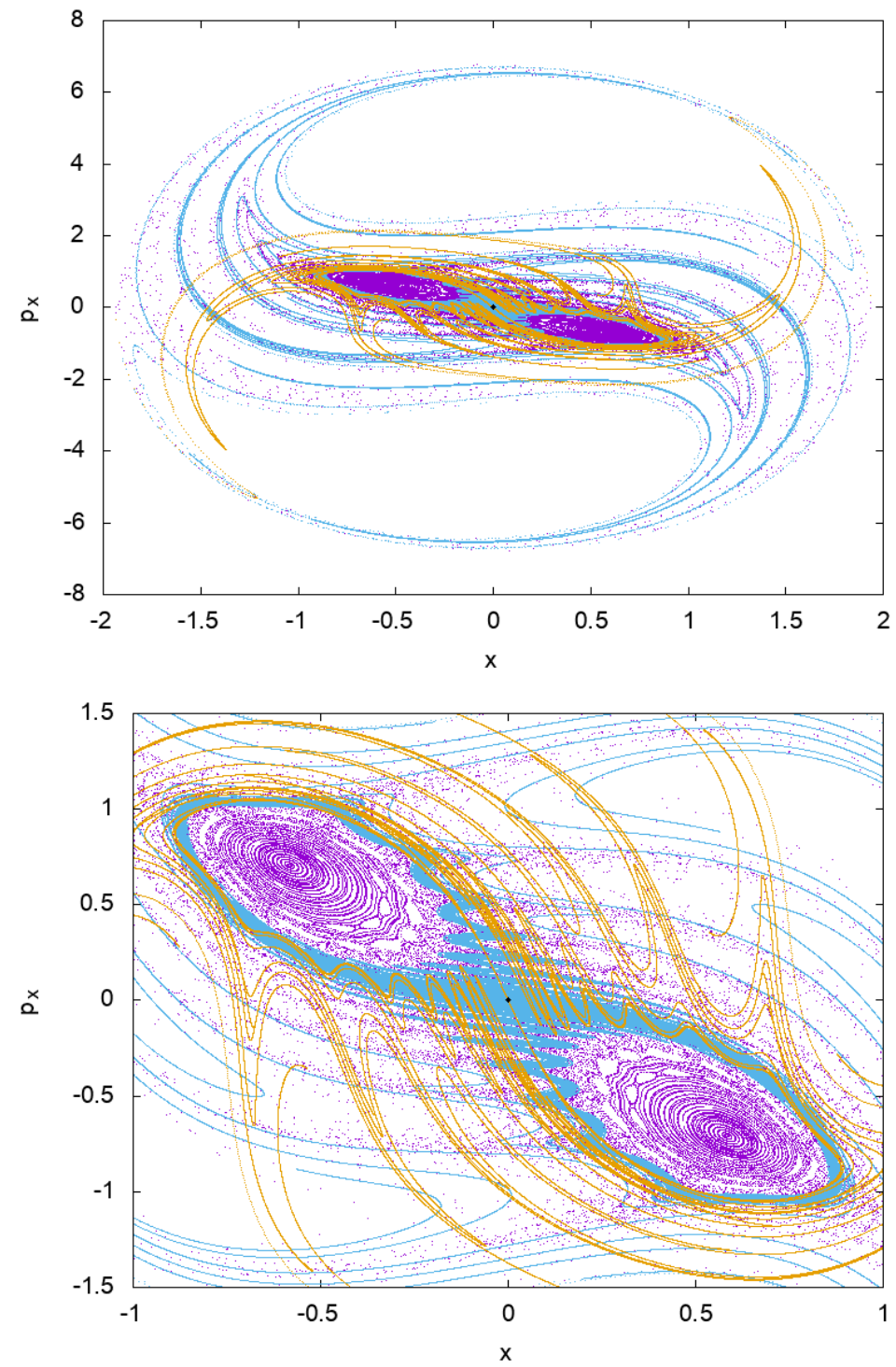

Fig. 11. Upper Panel: Poincaré surface of section $y=0$ with $p_{y}>0$ for $\mathrm{E}=23$. We observe invariant curves around the two points of the stable periodic orbit with period 2 of the bifurcation of the central minimum. We also observe the unstable (with cyan color) and stable (with orange color) invariant manifolds of the unstable periodic orbit of the family of the central minimum (the black point at the center). Lower Panel: An enlargement of the previous figure in order to see the central region around the periodic orbit of the central minimum and the invariant curves around the stable periodic orbit with period 2 of the bifurcation of the family of the central minimum

described in case I with the only difference that the unstable periodic orbit belongs to the family of the central minimum and it does not belong to a family of periodic orbits with period 10, as in case I.

We also study the phase space structure close to periodic orbits of the lower saddles. In Fig. 12 , we observe similar behaviour close to the neighbourhood of the periodic orbits of the lower saddles, on the Poincaré section $y=-1.8019693$ with $p_{y}>0$, with the behaviour that we encountered close to the neighbourhood of the periodic orbits of lower saddles in case I. The only difference is the central region. We have no invariant curves around the central periodic orbit of the central minimum as in fig. 10 , but we have invariant curves around the periodic orbit with period 2 of the bifurcation of the family of the central minimum (lower panel of the Fig. 12). We noticed the fact that the phase space at the neighbourhood of the periodic orbits of the lower saddles has not changed because these families have no bifurcations that would change the structure of the phase space in the neighbourhood of their periodic orbits. The change of the phase space happens at the central region which is in the neighbourhood of the periodic orbits of 

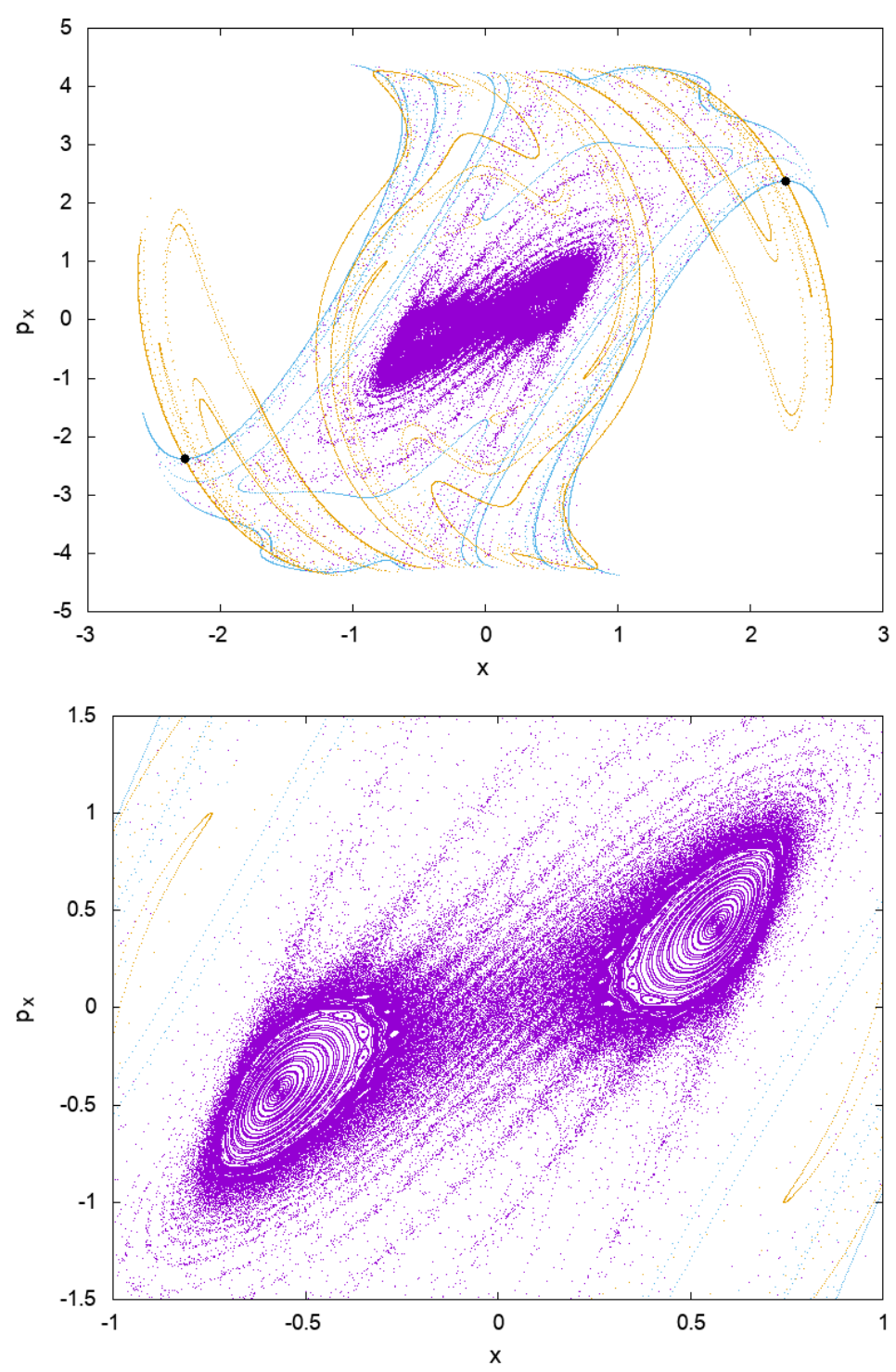

Fig. 12. Upper panel: Poincaré surface of section $y=-1.8019693$ with $p_{y}>0$ for $\mathrm{E}=23$. We see the unstable (cyan color) and stable (orange color) invariant manifolds of the periodic orbits of the two lower saddles, that are represented by two black points. Furthermore, we observe invariant curves around the stable periodic orbit with period 2 of the bifurcation of the family of the central minimum and scattered collection of points outside from them. Lower panel: An enlargement of the previous figure in order to see the central region around the periodic orbit of the central minimum and the invariant curves around the stable periodic orbit with period 2 of the bifurcation of the family of the central minimum.

the central minimum. This means that the mechanism of the entrance of the trajectories into the caldera does not change. It is the same as the corresponding mechanism in case I. The trajectories are trapped by the invariant manifolds of the periodic orbits of the lower saddles and they are guided by the unstable invariant manifolds close to the central region. Then they are trapped by the invariant manifolds of the periodic orbits of the family of the central minimum (Fig. 11) and they are transported from the stable manifolds to the center of the caldera.

\subsection{Case III}

In case III we have a similar situation as in case II with the difference being that we have the appearance of the families of periodic orbits of the upper saddles. In this subsection we study a representative example for $\mathrm{E}=29$. The trajectories that have initial conditions on the dividing surfaces of the periodic orbits of 

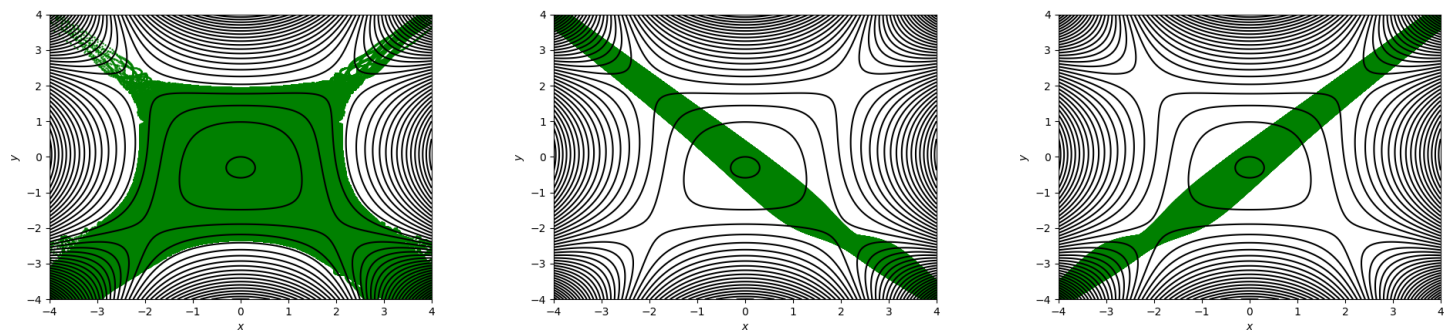

Fig. 13. Left Panel: Trajectories that have initial conditions on the dividing surface of a periodic orbit of the family of periodic orbits of the central minimum for $\mathrm{E}=29$ and contours of the potential energy. Central Panel: Trajectories that have initial conditions on the dividing surface of a periodic orbit of the family of periodic orbits of the upper left hand saddle for $\mathrm{E}=29$ and contours of the potential energy. Right Panel: Trajectories that have initial conditions on the dividing surface of a periodic orbit of the family of periodic orbits of the upper right hand saddle for $\mathrm{E}=29$ and contours of the potential energy.

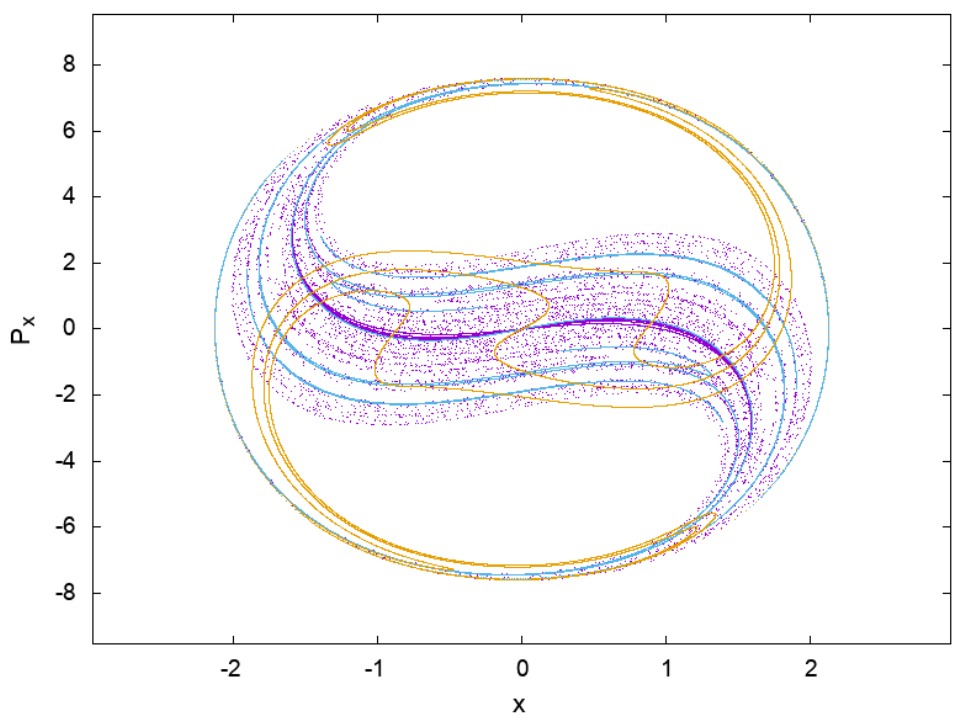

Fig. 14. Poincaré surface of section $y=0$ with $p_{y}>0$ for $\mathrm{E}=29$. We see the invariant manifolds (unstable with cyan color and stable with orange color) of the unstable periodic orbit of the family of the central minimum.

the central minimum are trapped for a long time at the central region of the caldera and then they exit from the four regions of the saddles (Fig. 13). The trajectories that have initial conditions on the dividing surfaces of the periodic orbits of the families of the upper saddles go straight across the caldera and exit via the opposite lower saddle (Fig. 13). This phenomenon is known as "dynamical matching" Collins et al., 2014.

The mechanism of the trapping of trajectories and exit from the caldera is the same as the corresponding mechanism in case II. As we can see in Fig. 14 the points of the trajectories are trapped inside the invariant manifolds of the periodic orbit of the family of the central minimum. This means that, as in case II, the invariant manifolds of the periodic orbits of the central minimum are responsible for the trapping and the transport of the trajectories to the exit from the caldera (through the regions of the four saddles - see Fig. 13 .

The mechanism of the entrance of the trajectories from the lower saddles to the central region of the caldera is the same as described in the previous subsections (cases I and II). This is because there is no change of the phase space structure at the neighbourhood of the periodic orbits of the families of the lower saddles. The trajectories that begin from the dividing surfaces of the periodic orbits of the families of the lower saddles have the same behaviour as that shown in the left panel of the Fig. 13. The only difference is that we have fewer trajectories than those of the left panel of the Fig. 13 to exit through the regions 


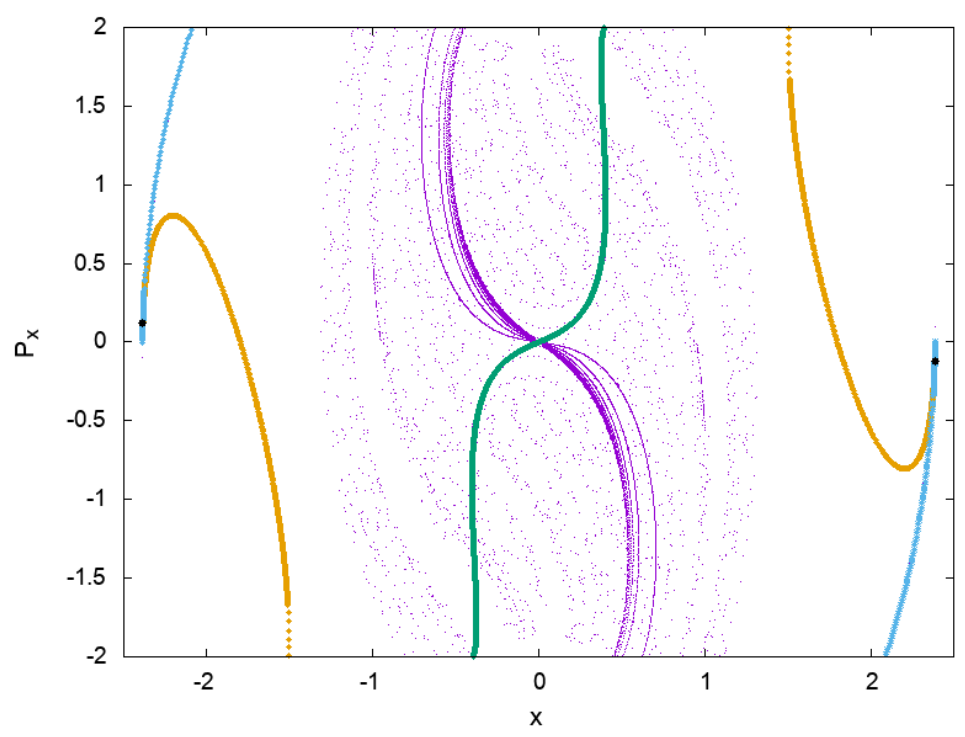

Fig. 15. Poincaré surface of section $y=1.884090$ with $p_{y}>0$ for $\mathrm{E}=29$. We see the unstable (cyan color) and stable (orange color) invariant manifolds of the periodic orbits of the two upper saddles, that are represented by two black points. Furthermore, we observe the invariant manifolds (unstable with violet color and stable with green color) of the periodic orbit of the family of the central minimum.

of the upper saddles. The reason is that the trajectories are trapped two times, one time by the invariant manifolds of the periodic orbits of the lower saddles and one time by the invariant manifolds of the periodic orbit of the family of the central minimum, for our time scale.

Next we study the phase space structure close to the periodic orbits of the families of the upper saddles using the Poincaré section $y=1.884090$ with $p_{y}>0$ (this is the section for which we can see these periodic orbits because they do not cross the plane $y=0$ ). We can observe in Fig. 15 that the central region has invariant manifolds of the periodic orbit of the family of the central minimum and scattering points around it. But the invariant manifolds of the periodic orbits of the families of the upper saddles are far away from the central region. The stable manifolds (with orange color in Fig. 15) bring trajectories at the neighbourhood of the periodic orbits that were far away from the central region and the unstable manifolds guide (with cyan color in Fig. 15) the points to infinity and far away from the central region. This means that we have no interaction of the invariant manifolds with the central region of the caldera and this explains the dynamical matching that we observe in the configuration space. Also the non-existence of the interaction of the invariant manifolds with the central region of the caldera explains the reason that we have fewer trajectories to leave from the upper saddles in the left panel of the Fig. 13. This happens because the trajectories that are trapped by the invariant manifolds of the unstable periodic orbit of the family of the central minimum can be trapped inside the invariant manifolds of the periodic orbits of the families of the lower saddles, as we can see in Figs. 10 and 12 for the cases I and II (this mechanism is the same for the case III). After this the trajectories are guided from the stable manifolds to the neighbourhood of the periodic orbits of the lower saddles. Moreover, the invariant manifolds of the periodic orbits of the upper saddles do not interact with the manifolds of the periodic orbits of the central region that are responsible for the transport of the trajectories to the region of the upper saddles.

\subsection{Case IV}

In case IV, we investigated a representative example for $\mathrm{E}=30$. In this case the periodic orbits of the family of the central minimum have become stable again, after the second bifurcation of the family of the central minimum. We have also a new family of unstable periodic orbits with period 2 that belongs to the bifurcation of the family of the central minimum. The trajectory behaviour of the orbits that have initial conditions on the dividing surfaces of the periodic orbits of the family of the central minimum and its bifurcation is the same as the trajectory behaviour that is represented in the left panel of the Fig. 13 in 

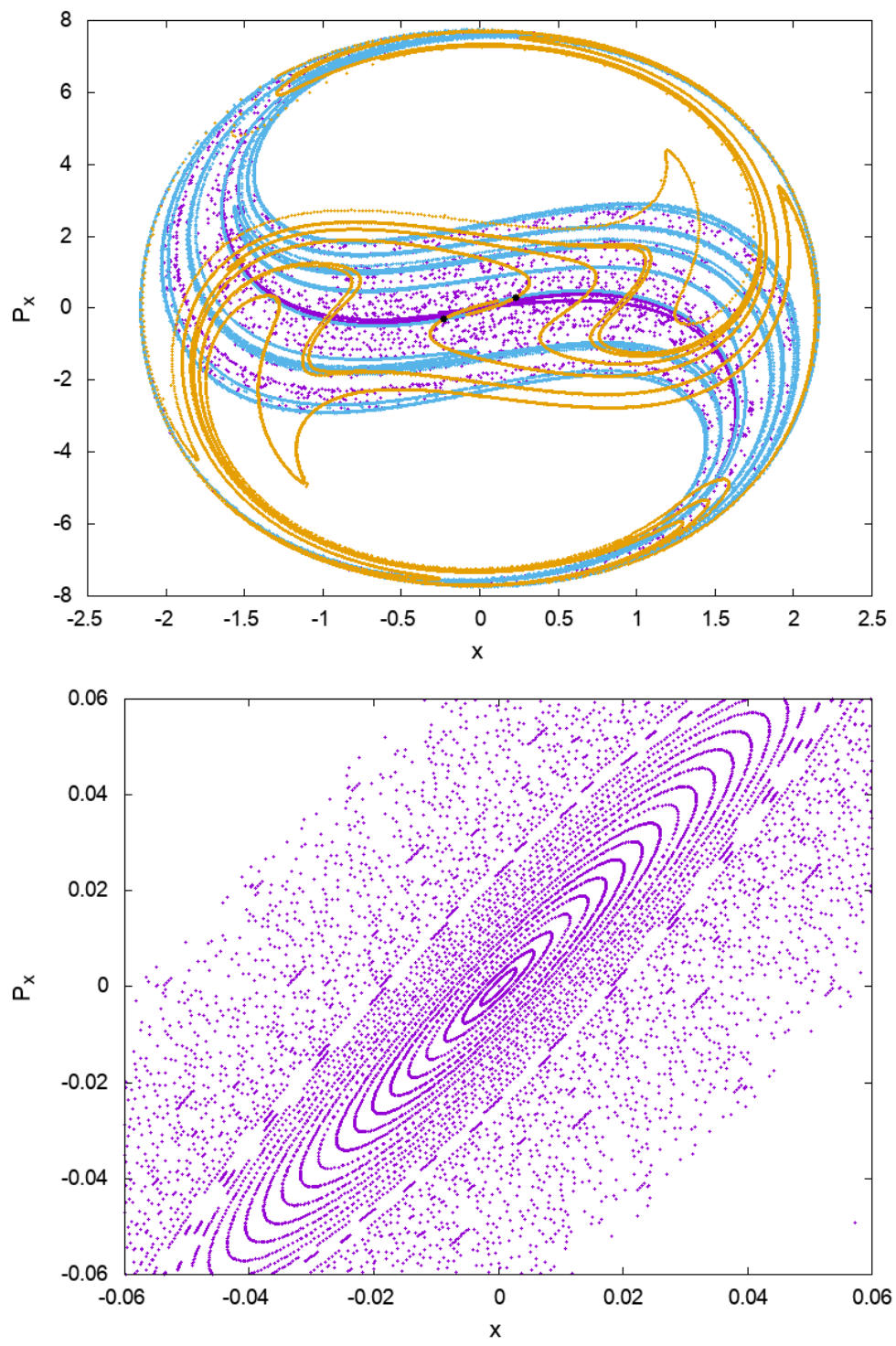

Fig. 16. Upper panel: Poincaré surface of section $y=0$ with $p_{y}>0$ for $\mathrm{E}=30$. We see the invariant manifolds (unstable with cyan color and stable with orange color) of the unstable periodic orbit with period 2 (that is represented by two black points) of the bifurcation of the family of the central minimum. Lower panel: An enlargement of the figure of the upper panel in order to see the invariant curves around the periodic orbit of the central minimum.

case III. The behaviour of trajectories that begin from the dividing surfaces of the periodic orbits of the families of the saddles is similar to that of the corresponding trajectories in case III.

We observe that the phase space structure close to the periodic orbit of the central minimum, on the Poincaré section $y=0$ with $p_{y}>0$ ( Fig. 16), is similar to the phase space structure close to the periodic orbit of the same family in case I. We observe invariant curves around the stable periodic orbit of the central minimum and many trajectories that are trapped by the invariant manifolds of the unstable periodic orbit with period 2 of the bifurcation of the family of the central minimum. The only difference is that we have now an unstable periodic orbit with period 2, while in case I we have an unstable periodic orbit with period 10. This means that the mechanism of trapping and guiding of the trajectories is the same as case I. The invariant manifolds of the unstable periodic orbit with period 2 are responsible for the trapping of trajectories in the central region of the caldera and their transport to the exit from the caldera.

The structure of the phase space in the neighbourhood of the periodic orbits of the families of the lower saddles has not changed. This is expected because we have no further bifurcation or other dynamical 
phenomenon for these families. This has as a result that the mechanism for the entrance into the caldera from the region of the lower saddles will be the same as for the previous cases I,II and III. This means that the trajectories are trapped by the invariant manifolds of the unstable periodic orbits of the lower saddles and they follow the unstable manifold of these periodic orbits close to the central region of the caldera. Then the trajectories are trapped again by the invariant manifolds of the unstable periodic orbits with period 2 of the bifurcation of the family of the central minimum and they follow the stable manifolds of these unstable periodic orbits to the central region of the caldera.

Similar to the case of the lower saddles, the phase space in the neighbourhood of the unstable periodic orbits of the upper saddles does not change. This means that the invariant manifolds do not interact with the central region of the caldera as in case III. This results in the 'dynamical matching' (see 3.3) of the trajectories that begin on the dividing surfaces of the periodic orbits of the families of upper saddles. 


\section{Summary and conclusions}

We studied the phase space structure and transport of the 2D caldera model. We encountered four different cases of the phase space structure. The phase space structure is determined by the transition of the stability of the family of periodic orbits of the central minimum from stability to instability and vice versa. Moreover, it is determined by the appearance of the new families of periodic orbits, that are bifurcations of the family of the central minimum, and the value of the energy. These cases are described in detail in 3 .

In this paper, we investigated the origin of the trajectory behaviour of the Caldera model and we found three mechanisms for this. These mechanisms are based on certain geometrical objects of the phase space, the invariant manifolds of the unstable periodic orbits and the KAM invariant tori. We saw that the geometry of the phase space give us important information for the trajectory behaviour that cannot be obtained from the study of the configuration space and the use of statistical methods. The mechanisms for the trajectory behaviour are:

(1) The trapping of trajectories and the entrance into the Caldera: The trajectories that have initial conditions on the dividing surfaces of the unstable periodic orbits of the low energy saddles are trapped inside the invariant manifolds of the unstable periodic orbits and they follow the unstable manifold (far away from the periodic orbits of the lower saddles), until they are trapped from the invariant manifolds of the unstable periodic orbits of the central region. The periodic orbits of the central region are, the unstable periodic orbits with period 10 that are outside the stable region of the stable periodic orbits of the family of the central minimum (case I), the unstable periodic orbits of the family of the central minimum (cases II and III) and the unstable periodic orbits with period 2 of a period-doubling bifurcation of the family of the central minimum (case IV). Then the trajectories follow the stable manifolds of these unstable periodic orbits towards to the central region of the caldera.

(2) The trapping of trajectories and the exit from the Caldera: The trajectories that have initial conditions at the central region of the potential are divided into two categories. In the first category, the trajectories are trapped for ever because they lie on or are inside the KAM invariant tori surround the stable periodic orbits that exist at the central region of the potential. In the second category, the trajectories are trapped inside the lobes of the invariant manifolds of the unstable periodic orbits of the central region (these periodic orbits are described in the first mechanism). The trajectories are transported from the unstable manifolds of these periodic orbits, via the regions of the saddles, to the exit from the caldera.

There is also a secondary mechanism that supports the basic mechanism of the exit from the caldera. In this secondary mechanism, many trajectories that are guided by the unstable manifolds of the periodic orbits of the central region are trapped inside the invariant manifolds of the unstable periodic orbits of the lower energy saddles. Afterwards, the trajectories follow the stable manifolds of the periodic orbits of the lower energy saddles, via the regions of the lower saddles, to the exit from the caldera. This secondary mechanism does not exist for the high energy saddles because the invariant manifolds of the unstable periodic orbits of the high energy saddles are not connected with the central region of the potential. This means that this secondary mechanism supports the exit of the trajectories from the caldera via the regions of the lower energy saddles. This is the reason why the majority of the trajectories exit from the regions of the lower energy saddles.

(3) Dynamical matching: The trajectories that have initial conditions on the dividing surfaces of the periodic orbits of the high energy saddles go straight across the caldera and exit via the opposite lower saddle (dynamical matching [Collins et al., 2014]). This behaviour is due to the fact that the invariant manifolds of the unstable periodic orbits of the high energy saddles do not interact with the central region of the caldera. The trajectories follow the invariant manifolds far away from the central region of the caldera, without any interaction with it.

Although the phase space structures described in this paper were derived for a 2D potential, the experimental observations for the reactions cited in the introduction suggest that broadly similar objects may exist in the high dimensional phase spaces representing the reactions of polyatomic molecules occurring on caldera-type potentials. 


\section{Acknowledgments}

We acknowledge the support of EPSRC Grant No. EP/P021123/1 and ONR Grant No. N00014-01-10769.

\section{References}

Arnold, V. [1963] " Proof of an Kolmogorov's theorem on the conservation of conditionally periodic motions with a small variation in the hamiltonian." Russian Math. Surv. 18, 9 -36.

Arnold, V. [1978] Mathematical methods of classical mechanics, (vol. 60 Graduate Texts in Mathematics, Springer)

Baldwin, J. [2003] "Thermal rearrangements of vinylcyclopropanes to cyclopentenes." Chemical reviews 103(4), 1197-1212 .

Birkhoff, G.D. [1960] Dynamical systems. American Mathematical Society.

Carpenter, B., Ezra, G., Farantos, S., Kramer, Z., Wiggins, S. [2018] "Dynamics on the double morse potential: A paradigm for roaming reactions with no saddle points." Regular and Chaotic Dynamics 23(1), 60-79.

Carpenter, B., Harvey, J., Orr-Ewing, A. [2016] " The study of reactive intermediates in condensed phases." Journal of the American Chemical Society 138(14), 4695-4705.

Collins, P., Kramer, Z., Carpenter, B., Ezra, G., Wiggins, S. [2014] "Nonstatistical dynamics on the caldera." Journal of Chemical Physics 141(034111).

Contopoulos, G. [2002] Order and chaos in dynamical astronomy. (Springer Verlag)

Doering, W.v.E., Cheng, X., Lee, K., Lin, Z. [2002] "Fate of the intermediate diradicals in the caldera: stereochemistry of thermal stereomutations, $(2+2)$ cycloreversions, and $(2+4)$ ring-enlargements of cis- and trans- 1-cyano-2-(eandz)-propenyl-cis-3,4-dideuteriocyclobutanes." Journal of the American Chemical Society 124(39), 11642-11652.

Doering, W.v.E., Gilbert, C., Leermakers, P.: Symmetrical distribution of energy in initially unsymmetrically excited products : Reaction of dideuteriodiazomethane with allene, methylenecyclopropane and vinylcyclopropane. Tetrahedron 24, 6863-6872.

Doubleday, C., Bolton, K., Hase, W. [1997] Direct dynamics study of the stereomutation of cyclopropane Journal of the American Chemical Society 119 (22), 5251-5252.

Doubleday, C., Nendel, M., Houk, K., Thweatt, D., Page, M. [1999] "Direct dynamics quasiclassical trajectory study of the stereochemistry of the vinylcyclopropane - cyclopentene rearrangement." Journal of the American Chemical Society 121(19), 4720-4721.

Doubleday, C., Suhrada, C., Houk, K. [2006] " Dynamics of the degenerate rearrangement of bicyclo[3.1.0]hex-2-ene" Journal of the American Chemical Society 128(1), 90-94.

Goldschmidt, Z., Crammer, B. [1988] "Vinylcyclopropane rearrangements." Chem. Soc. Rev 17, $229-267$.

Kolmogorov, A. [1954] "On the conservation of conditionally periodic motions under small perturbation of the hamiltonian." Dokl. Akad. Nauk SSSR, vol. 98, pp. 527-530.

Mauguiere, F., Collins, P., Kramer, Z., Carpenter, B., Ezra, G., Farantos, S., Wiggins, S. [2016] "Phase space barriers and dividing surfaces in the absence of critical points of the potential energy: Application to roaming in ozone." Journal of Chemical Physics 144 (054107).

Moser, J. [1962] "On invariant curves of area-preserving mappings of an annulus." Nachr. Akad. Wiss. Göttingen, II pp. 1-20 .

Moser, J. [1976] "Periodic orbits near an equilibrium and a theorem by alan weinstein." Communications on Pure and Applied Mathematics 29(6), 727-747.

Poincaré, H. [1892] Les méthodes nouvelles de la mécanique céleste. (Gauthier-Villars, Paris).

Rabinowitz, P. [1982] Periodic solutions of hamiltonian systems: a survey. SIAM J. Math. Anal. 13(3), 343-352.

Reyes, M., Lobkovsky, E., Carpenter, B. [2002] "Interplay of orbital symmetry and nonstatistical dynamics in the thermal rearrangements of bicyclo[n.1.0]polyenes." $J$ Am Chem Soc 124, 641-651.

Weinstein, A. [1973] "Normal modes for nonlinear hamiltonian systems." Inventiones mathematicae 20(1), $47-57$. 
Wiggins, S. [2003] Introduction to applied nonlinear dynamical systems and chaos, (2 edn. Springer Science \& Business Media).

\section{Appendix A Linear stability and bifurcations of periodic orbits}

We present the method of the computation of the linear stability of periodic orbits. The 2D Poincaré map of a Hamiltonian system of two degrees of freedom (like what is considered in this paper) is given by equations of the form :

$$
\begin{array}{r}
x_{1}=f_{1}\left(x_{0}, p_{x_{0}}\right) \\
p_{x_{1}}=f_{2}\left(x_{0}, p_{x_{0}}\right) \\
\vdots \\
x_{n}=f_{1}^{n}\left(x_{0}, p_{x_{0}}\right) \\
p_{x_{n}}=f_{2}^{n}\left(x_{0}, p_{x_{0}}\right)
\end{array}
$$

For a periodic orbit with period $\mathrm{n}$ we have $x_{n}=x_{0}$ and $p_{x_{n}}=p_{x_{0}}$. The functions $f_{1}$ and $f_{2}$ are obtained by numerical integration of an trajectory with initial conditions on the Poincaré section until its first return to the Poincaré section. The functions $f_{1}^{n}$ and $f_{2}^{n}$ are obtained by numerical integration of an trajectory with initial conditions on the Poincaré section until its nth return to the Poincaré section.

We have two types of periodic orbits, according to their linear stability: stable and unstable periodic orbits. The numerical calculation of the linear stability of periodic orbits in 2D autonomous Hamiltonian systems is based on the method of Hénon (see e.g. [Contopoulos, 2002]). We first consider small deviations from the initial conditions of the periodic orbit on the 2D Poincaré surface of section and then integrate the trajectory again to the next intersection. The relation of the final deviations of this neighbouring trajectory (after the first return to the Poincaré surface of section) from the periodic orbit, with the initially introduced deviations can be written in vector form as $\xi=M \xi_{0}$, where $\xi$ is the final deviation (after the first return to the Poincaré surface of section) and $\xi_{0}$ is the initial deviation, $\mathrm{M}$ is a $2 \times 2$ matrix, called the monodromy matrix.

$$
M=\left[\begin{array}{ll}
a & b \\
c & d
\end{array}\right]
$$

This matrix satisfies the symplectic identity Arnold, 1978]: $M^{T} J M=J$ where $M^{T}$ denotes the transpose of $M$ and $J$ is the fixed nonsingular, skew-symmetric $2 \times 2$ matrix:

$$
J=\left[\begin{array}{cc}
0 & 1 \\
-1 & 0
\end{array}\right]
$$

$J$ satisfies the relation $J^{-1}=J^{T}=-J$, where $J^{T}$ and $J^{-1}$ are the transpose and the inverse of matrix $J$, respectively. The symplectic identity in our case (in which we have a symplectic matrix $2 \times 2$ ) is equivalent with the condition $\operatorname{Det} M=1$ (where Det denotes the determinant of a matrix), $M^{T} J M=J \Leftrightarrow \operatorname{Det} M=1$ (this is not true for the general case of a symplectic matrix $M$, that is a $2 n \times 2 n$ matrix with $n \in \mathbb{N}_{\neq 0}$, for $n>1)$. The elements of the monodromy matrix $M$ are:

$$
\begin{aligned}
a & =\frac{\partial f_{1}}{\partial x}\left(x_{0}, p_{x_{0}}\right) \\
b & =\frac{\partial f_{1}}{\partial p_{x}}\left(x_{0}, p_{x_{0}}\right) \\
c & =\frac{\partial f_{2}}{\partial x}\left(x_{0}, p_{x_{0}}\right) \\
d & =\frac{\partial f_{2}}{\partial p_{x}}\left(x_{0}, p_{x_{0}}\right)
\end{aligned}
$$


It can be shown that the characteristic equation of $M$ is $\lambda^{2}-(a+d) \lambda+1=0$ and the eigenvalues $\lambda_{1}, \lambda_{2}$ are the roots of this equation. We define the quantity $\alpha=|a+d| / 2$ to be the Hénon stability parameter (see e.q. Contopoulos, 2002]). We have two kinds of periodic orbits:

(1) Stable periodic orbits: In this case $\alpha<1$ and the eigenvalues of the monodromy matrix of a 2D Poincaré map are complex conjugate and they are on the unit circle.

(2) Unstable periodic orbits: In this case $\alpha>1$ and the eigenvalues of the monodromy matrix of a 2D Poincaré map are real and outside of the unit circle.

A family of periodic orbits can be stable or unstable if its periodic orbits are stable or unstable, respectively. The eigenvalues of the periodic orbits move on the unit circle (for stable families - Fig. A.1) or on the real axis (for unstable families - Fig. A.1) as a parameter of the system varies (in our case the parameter is the value of the Energy). If the eigenvalues meet each other at the points $\lambda=1$ and $\lambda=-1$ we have a collision. After this collision, the eigenvalues either move on the real axis (if they were on the unit circle before collision) or on the unit circle (if they were on the real axis before collision). This means that we have a transition of this family from stability to instability or vice versa. If the collision is at the point $\lambda=1$ we have the appearance of one or two new families of periodic orbits (pos) with equal period. If the collision is at the point $\lambda=-1$ we have the appearance of two new families of periodic orbits (pos) with twice the period. These families are referred to as bifurcations of the mother family (the initial family). There are three types of bifurcations of equal period and doubled period. We will describe all these types of bifurcations using the characteristic. The characteristic is a curve that gives the initial conditions ( $\mathrm{x}$ or $p_{x}$ for our case) of the periodic orbits of a family, on the Poincaré surface of section, as a function of a parameter of the system (Energy E in our case). The three types of bifurcations are:

(1) Saddle-node Bifurcation : In this case, the characteristic has a minimum or maximum ( for example the minimum at $\mathrm{K}$ in Fig. A.2). The one branch of the characteristic represents a stable family (for example the family f in Fig. A.2 and the other represents an unstable family (for example the family g in Fig. A.2p.

(2) Pitchfork Bifurcation (supercritical or subcritical) : In this case, we have two new symmetric families of equal period ( $\mathrm{g}$ and $\mathrm{h}$ in Fig. A.3), that are born at the bifurcation point (in our example point K - see Fig. A.3) and they exist on the side of the unstable part of the initial family (family $\mathrm{f}$ in Fig. A.3) in the supercritical case ( see left panel of Fig. A.3) or on the side of the stable part of the initial family (family f in Fig. A.3) in the subcritical case (see right panel of Fig. A.3.

(3) Period-doubling Bifurcation (supercritical or subcritical) : In this case, we have two symmetric arc of a new family of double period (g1 and g2 in Fig. A.4), that are generated at the bifurcation point (in our example point K - see Fig. A.4) and they exist on the side of the unstable part of the initial family (family f in Fig. A.4) in the supercritical case ( see left panel of Fig. A.4) or on the side of the stable part of the initial family (family $\mathrm{f}$ in Fig. A.4) in the subcritical case (see right panel of Fig. A.4.

In rare cases we have bifurcations of families of equal or doubled period, that are similar to the Pitchfork Bifurcation or Period-doubling Bifurcation [Contopoulos, 2002]. The only difference is that the new families are asymmetric. This means that the two characteristics of the new families of equal period are not symmetric to each other or the characteristic of the new family of doubled period has not two symmetric arc. These bifurcations are referred to as asymmetric bifurcations of equal period (supercritical or subcritical) and period-doubling asymmetric bifurcations (supercritical or subcritical). 


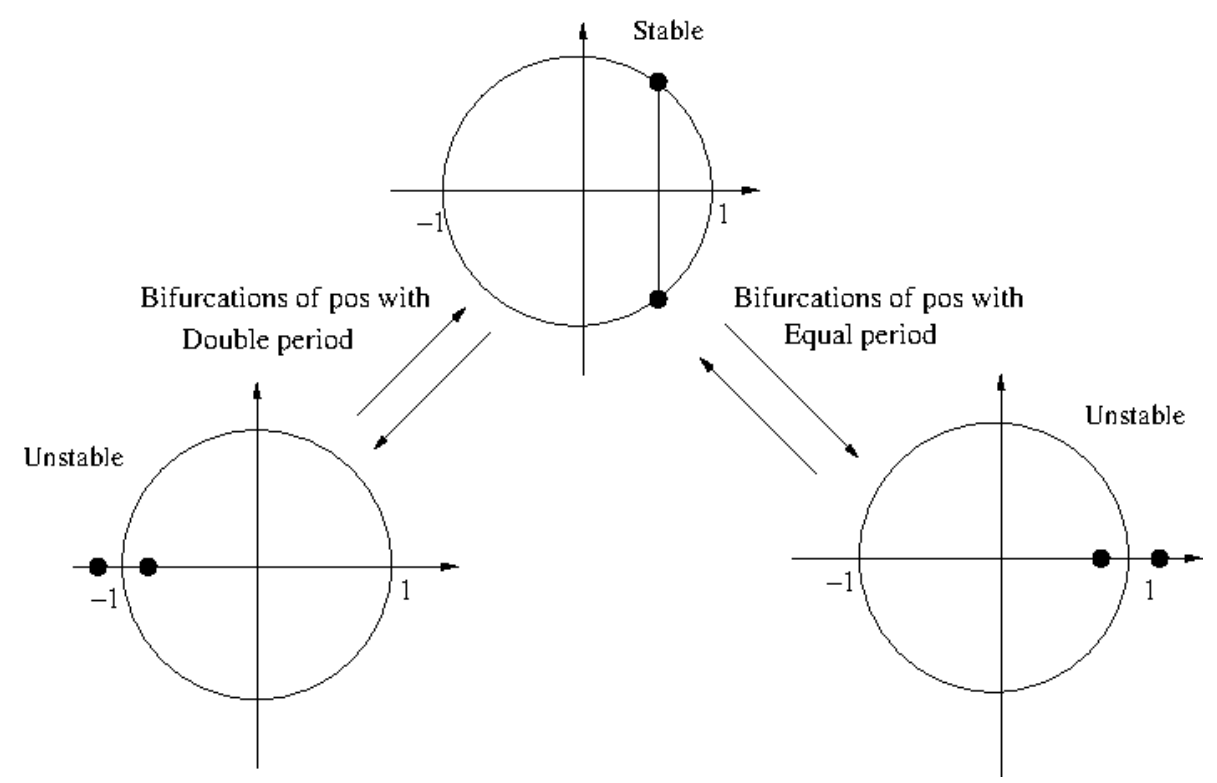

Fig. A.1. The Eigenvalues move on the unit circle (stable periodic orbit) and collide at the points $\lambda=1$ and $\lambda=-1$, then the eigenvalues move on the real axis (unstable periodic orbit) and vice versa. The result of the collision of the eigenvalues at the points $\lambda=1$ and $\lambda=-1$ is that new families of periodic orbits (pos) with equal period and with double period appear in the system respectively.

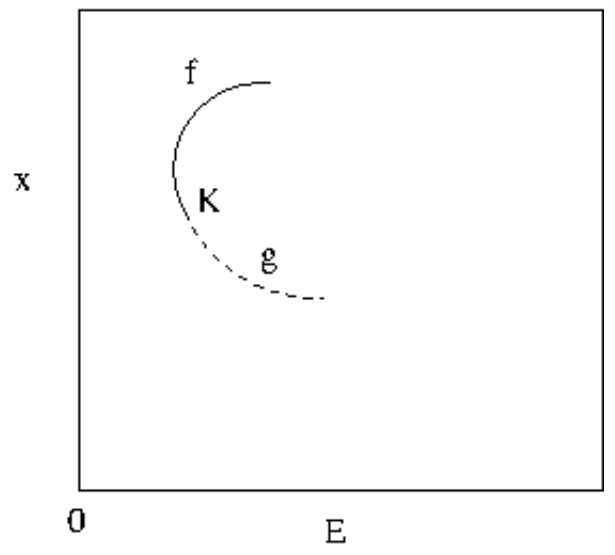

Fig. A.2. Characteristic near a saddle-node bifurcation at K. The solid and dashed lines depict the stable and unstable parts of the families of periodic orbits.
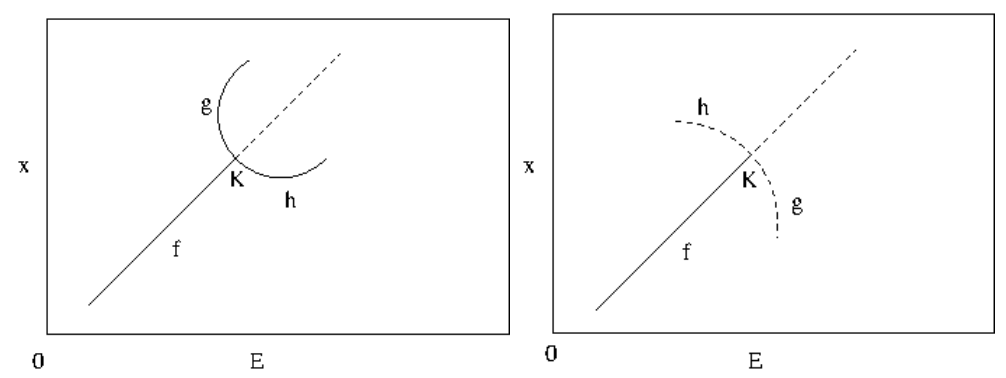

Fig. A.3. Characteristic near a pitchfork bifurcation at K. The solid and dashed lines depict the stable and unstable parts of the families of periodic orbits. Left panel: Supercritical pitchfork bifurcation. Right panel: Subcritical pitchfork bifurcation. 

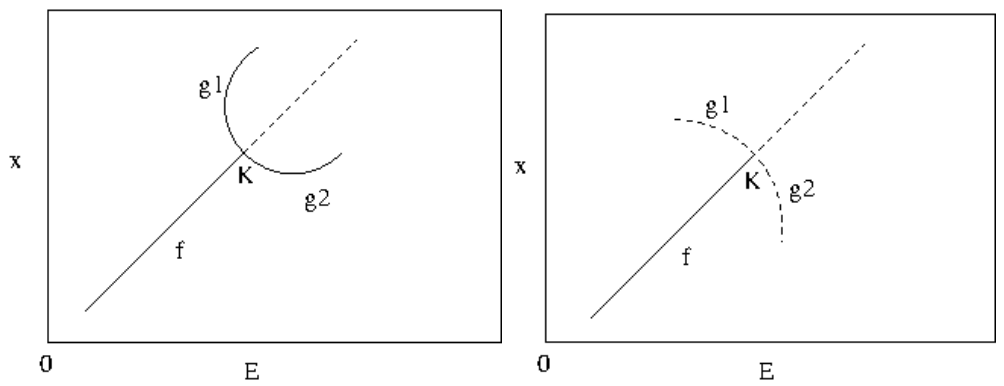

Fig. A.4. Characteristic near a period-doubling bifurcation at K. The solid and dashed lines depict the stable and unstable parts of the families of periodic orbits. Left panel: Supercritical period-doubling bifurcation. Right panel: Subcritical perioddoubling bifurcation. 


\section{Appendix B Dividing Surfaces}

In this paper we use the notion of the dividing surface (DS). A dividing surface is a surface of one less dimension than the energy surface. A dividing surfaces is a 2D surface (with fixed total Energy) where the periodic orbit forms the 1D boundary of the dividing surface. We define the dividing surface in order to divide a region of interest, according the topography of the potential. In this paper, the dividing surfaces are used to monitor the exit and the entrance of the trajectories to the regions of the periodic orbits of the families of four saddles, that are connected with the entrance into and exit from the caldera. Moreover we use the dividing surface in order to monitor the entrance into and exit from the region of periodic orbits of the families of the central minimum and its bifurcations and also to the regions of periodic orbits with period 10 that are in the central region of the potential. The main reason we choose these dividing surfaces is that we want to study, firstly the behaviour of the trajectories when entering and exiting the caldera, and secondly the behaviour of the trajectories when entering to and leaving from the central area of the caldera. This will help us to understand and categorize the different possible paths followed by the trajectories from the central area to the entrance and exit areas and vice versa.

The algorithm for the construction of a dividing surface (for fixed total energy E) is given in Carpenter et al. 2018]. This provides a discrete version of a $2 \mathrm{D}$ surface having the no-recrossing property and minimal flux |Mauguiere et al., 2016]:

(1) Locate a periodic orbit.

(2) Project the periodic orbit into configuration space.

(3) Choose points on that curve $\left(x_{i}, y_{i}\right)$ for $i=1, \ldots N$ where $N$ is the desired number of points. Points are spaced uniformly according to distance along the periodic orbit.

(4) For each point $\left(x_{i}, y_{i}\right)$ determine $p_{x \max , i}$ by solving:

$$
H\left(x_{i}, y_{i}, p_{x}, 0\right)=\frac{p_{x}^{2}}{2 m}+V\left(x_{i}, y_{i}\right)
$$

for $p_{x}$. Note that solution of this equation requires $E-V\left(x_{i}, y_{i}\right) \geq 0$ and there will be two solutions, $\pm p_{x \max , i}$.

(5) For each point $\left(x_{i}, y_{i}\right)$ choose points for $j=1, \ldots, K$ with $p_{x_{1}}=0$ and $p_{x_{K}}=p_{x m a x, i}$ and solve the equation $H\left(x_{i}, y_{i}, p_{x}, p_{y}\right)=E$ to obtain $p_{y}$.

The time of integration of the trajectories, on dividing surfaces, was 5 time units that is enough to see all the different kinds of trajectory behaviour Collins et al., 2014. 